\title{
TỰ ĐộNG TÁCH CHIẾT CÁC YẾU TỐ DẠNG TUYẾN TƯ ẢNH SPOT KHU VỰC TỈNH BĂ KẠN
}

\author{
ThS. NGUYẼN ĐìNH TÀl, PGS. TS. NGUYẼN NGỌC THẠCH \\ Khoa Địa lý, Trường Đại học Khoa học Tụ̂ nhiên, ĐHQGHN
}

\section{Tóm tắt:}

Nghiên cứu cấu trúc của các yếu tố dạng tuyến (lineament) là một nội dung quan trọng trong các nghiên cứu về kiến tạo địa chất, thăm dò khoáng sản và đánh giá nguy cơ trượt lở. Nghiên cứu này đề cập đến việc tách chiết các đối tượng dạng tuyến từ ảnh vệ tinh SPOT cũng như phân tích các lineament đó. Các kỹ thuật tăng cường chất lượng ảnh đã được sử dụng để nâng cao khả năng phát hiện các lineament theo các hướng khác nhau. Module LINE của phần mềm PCI Geomatica được sử dụng để tự động tách chiết các lineament theo các thông số định trước. Việc chuẩn hóa các lineament, cũng như loại bỏ các lineament giả đã được chúng tôi sử dụng để nâng cao độ chính xác của bản đồ kết quả thông qua 2 kỹ thuật tự động và bằng tay. Các thống kê dựa trên bản đồ lineament, đã phản ánh phương pháp tách chiết lineament áp dụng cho khu vực Bắc Kạn phù hợp với hướng cấu trúc địa hình khu vực nghiên cứu.

\section{Mở đầu}

Lineament có thể phản ánh bề mặt của sự gián đoạn trong những tảng đá hoặc có thể phản ánh các cấu trúc địa chất, đặc điểm địa hình hoặc các đặc điểm con người gây ra. Lineament có thể dưới dạng đường thẳng hoặc hơi cong (O'Leary và nnk., 1976). Chiết xuất lineament từ ảnh vệ tinh là rất quan trọng cho nhiều mục đích như bản đồ địa chất và thăm dò khoáng sản (Rowan và Lathram, 1980) và đánh giá nguy cơ động đất và sạt lở đất (Stefouli và nnk., 1996). Lineament thường được tách chiết và giải đoán từ ảnh vệ tinh bằng tay hoặc tự động.

Trong những năm gần đây, hầu hết các phương pháp tách chiết đều dựa trên giải đoán ảnh bằng mắt bởi chuyên gia hoặc tự động phát hiện từ ảnh vệ tinh. Các phương pháp tự động tiết kiệm thời gian và cải thiện tính khách quan quá trình tách chiết lineament. Nhiều tác giả khác đã giải đoán cấu trúc lineament từ ảnh vệ tinh bằng cách sử dụng kỹ thuật tự động tách chiết (Costa và Starkey, 2001; Mostafa và Bishta, 2005).

\section{Phương pháp nghiên cứu}

\subsection{Tăng cường chất lượng ảnh}

Một trong những tính năng đặc trưng của ảnh vệ tinh là thông số tần suất không gian được định nghĩa là số lượng các thay đổi trong giá trị độ sáng trên một đơn vị khoảng cách cho bất bỳ một phần cụ thể nào của ảnh. Nếu có rất ít thay đổi trong giá trị độ sáng trong một khu vực nhất định trên ảnh, thì được gọi là khu vực có tần số thấp. Ngược lại, nếu giá trị độ sáng thay đổi đáng kể trong khoảng cách ngắn, đây là khu vực có tần số cao (Jensen, 1996). Vì vậy, hoạt động lọc được sử dụng để tăng cường hoặc 
làm giảm bớt tần số không gian trong ảnh. Tần số này có thể là do sự có mặt của các lineament trong khu vực. Nói cách khác, hoạt động lọc sẽ làm sắc nét ranh giới tồn tại giữa các đơn vị liền kề (Sarp, 2005). Trong nghiên cứu này, lọc định hướng đã được sử dụng để tăng cường, tách chiết, và phân loại hướng lineament. Hướng được chọn phụ thuộc và cấu trúc và kiến tạo của khu vực, tuy nhiên bộ lọc định hướng được áp dụng cho ảnh SPOT sử dụng quá trình chập bởi cửa sổ với (3×3) pixel theo bộ lọc hạt nhân Sobel (Xem bảng 1). Đây là bộ lọc theo 4 hướng chính, giúp tăng cường khả năng giải đoán, tách chiết các đối tượng lineament (Süzen và Toprak, 1998). Việc lọc theo các hướng được thực hiện bằng phần mềm ERDAS 9.2. (Xem hình 1)

\subsection{Tự động tách chiết các đối tượng dạng tuyến}

Các ảnh vệ tinh sau khi đã được lọc theo 4 hướng, lần lượt được tách chiết tự động các lineament thông qua module LINE của phần mềm $\mathrm{PCl}$ Geomatica $(\mathrm{PCl}, 2010)$. Đây là một modul chuyên dụng cho việc triết xuất lineament từ ảnh viễn thám. Việc tách chiết lineament được tiến hành theo các thông số tương tự được tối ưu hóa (Mostafa và Bishta, 2005) cho khu vực nghiên cứu.

Những thông số là:

1. Edge filter radius=3

2. Minimum edge gradient $=15$

3. Minimum line length $=15$

4. Line fitting tolerance $=2$

5. Maximum angular difference $=10$

6. Maximum linking distance $=30$

Các kết quả thu được đó là 4 file lineament ở dạng vector (xem hình 2). Các file lineament này sau đó được gộp lại thành 1 file lineament. Đây có thể coi là một sơ đồ lineament ở dạng thô.

\subsection{Chuẩn hóa Lineament}

Thông thường các lineament được mô tả trong các sơ đồ, bản đồ dưới dạng các đoạn thẳng hoặc đường thẳng và chiều dày của các lineament hầu như không được tính đến. Tuy nhiên trên thực tế các lineament cũng có độ dày, ví dụ như độ rộng của đứt gãy, đới phá hủy... Bên cạnh đó, trên ảnh viễn thám không phải bao giờ độ rộng của các lineament cũng là 1 pixel ảnh. Chính vì vậy sau quá trình lọc lineament theo 4 hướng khác nhau, rất nhiều lineament thực tế chỉ là 1 đường nhưng sau quá trình lọc có thể thành nhiều đường và lệch nhau một góc nhất định. Cũng có trường hợp chúng tịnh tiến đi một khoảng nhất định tùy thuộc vào độ rộng của các lineament và đứt gãy. Do vậy các lineament đó cần được loại bỏ và tối giản sao cho chỉ có một lineament duy nhất mà thôi. Ngoài ra có nhiều lineament quá ngắn không có nhiều ý nghĩa trong nghiên cứu cũng cần lọc ra để loại bỏ. Bên cạnh đó nhiều lineament gần nhau có thể nối lại thành một lineament lớn. Thêm nữa, một số lineament được tạo ra do module tách chiết không thể nhận biết được đâu là lineament trên ảnh do con người tạo ra như đường xá, cầu cống...Đây được coi là các lineament giả và cũng cần phải lọc bỏ. Quá trình chuẩn hóa lineament được tiến hành bằng 2 phương pháp:

\subsubsection{Chuẩn hóa tự động}

Một số kỹ thuật chuẩn hóa lineament tự động đã được trình bày trong nghiên cứu của (Hung, L. Q, và nnk., 2005). Quá trình chuẩn hóa lineament hoàn toàn tự động dựa trên thống kê các lineament được tách 
Bảng 1: Bốn hướng chính của bộ lọc Sobel

\begin{tabular}{|c|c|c||c|c|c||c|c|c||c|c|c|}
\hline \multicolumn{3}{|c|}{ Bắc - Nam } & \multicolumn{3}{|c|}{ Đông Bắc -Tày Nam } & \multicolumn{3}{|c|}{ Đông - Tây } & \multicolumn{3}{|c|}{ Tây Băc -Đông Nam } \\
\hline \hline-1 & 0 & 1 & -2 & -1 & 0 & -1 & -2 & -1 & 0 & 1 & 2 \\
\hline-2 & 0 & 2 & -1 & 0 & 1 & 0 & 0 & 0 & -1 & 0 & 1 \\
\hline-1 & 0 & 1 & 0 & 1 & 2 & 1 & 2 & 1 & -2 & -1 & 0 \\
\hline
\end{tabular}

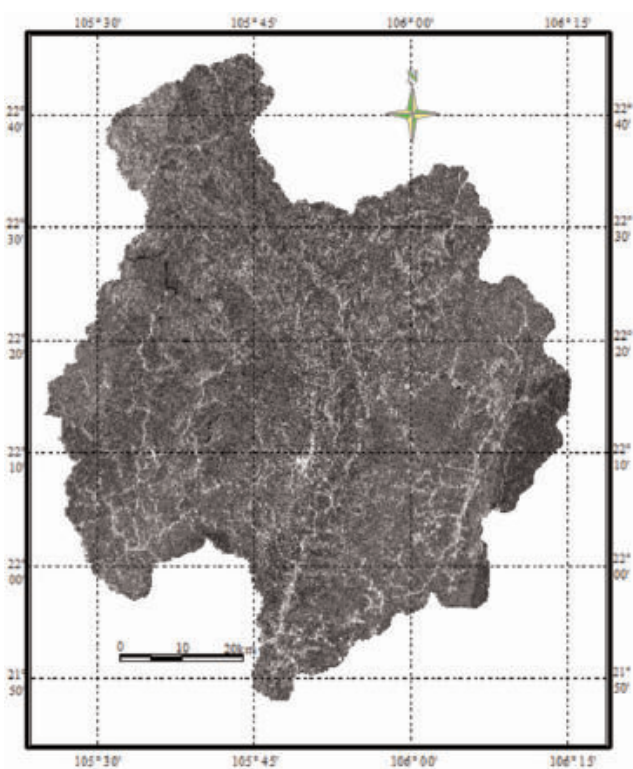

Hướng $B-N$

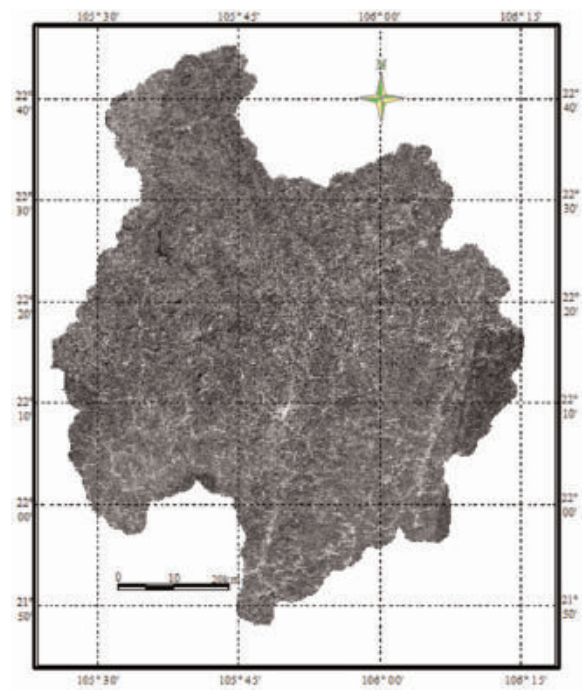

Hướng $Đ-T$

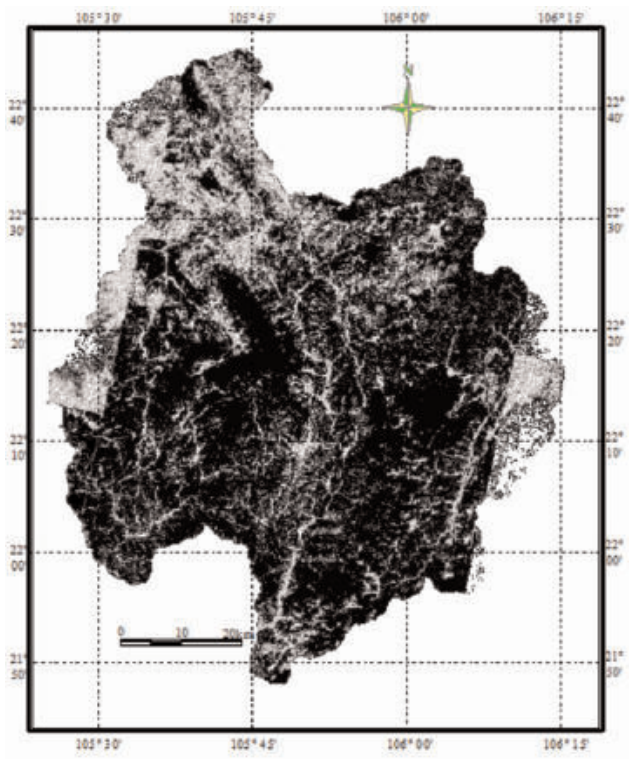

Hướng $Đ B-T N$

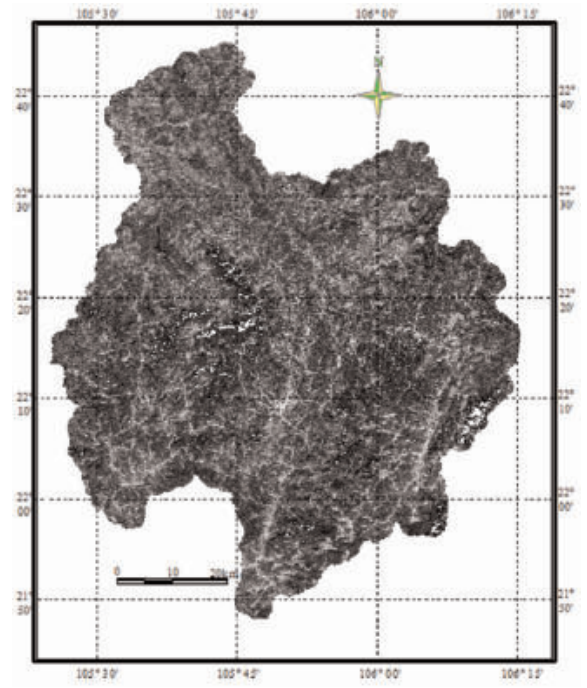

Hướng TB-ĐN

Hình 1: Các ảnh đã được nâng cao chất lượng sau khi sử dụng các bộ lọc 


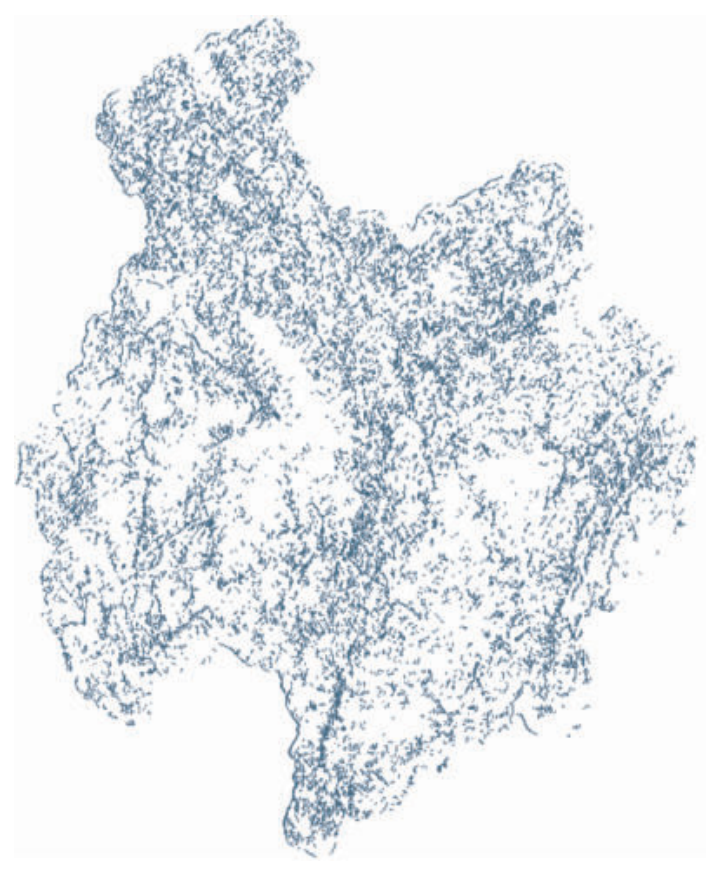

Hướng B-N

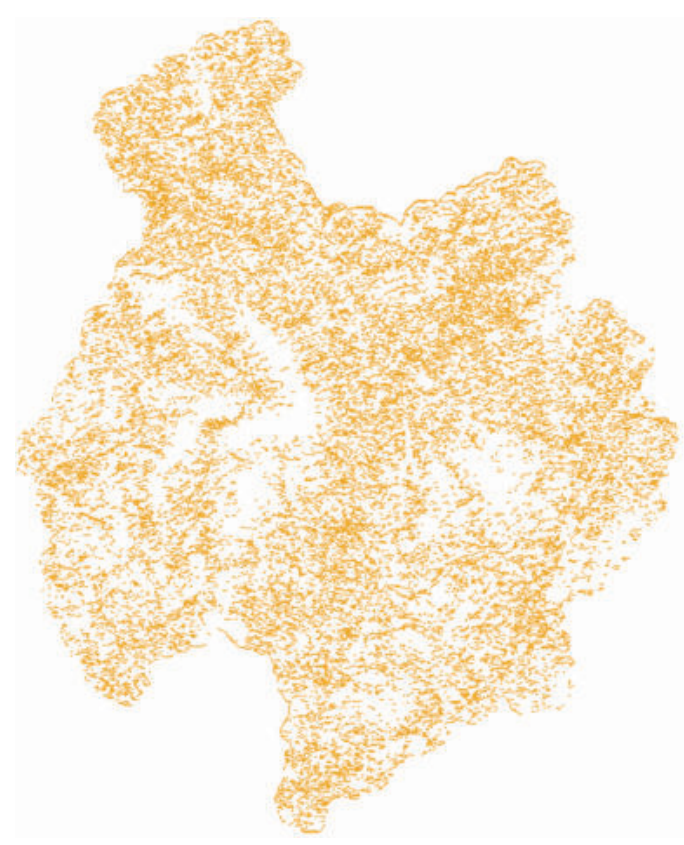

Hướng $\bigoplus-T$

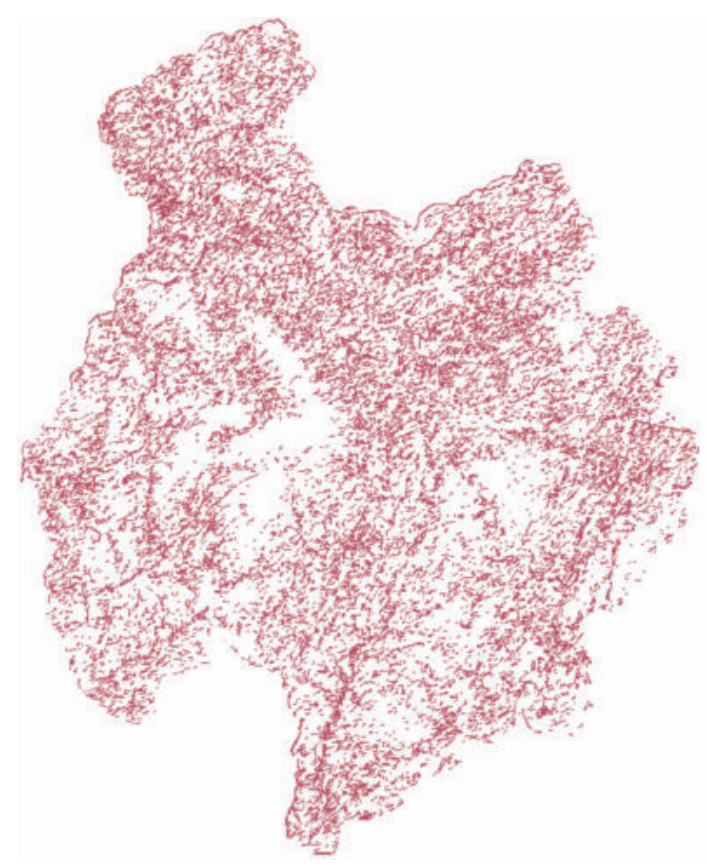

Hướng $Đ B-T N$

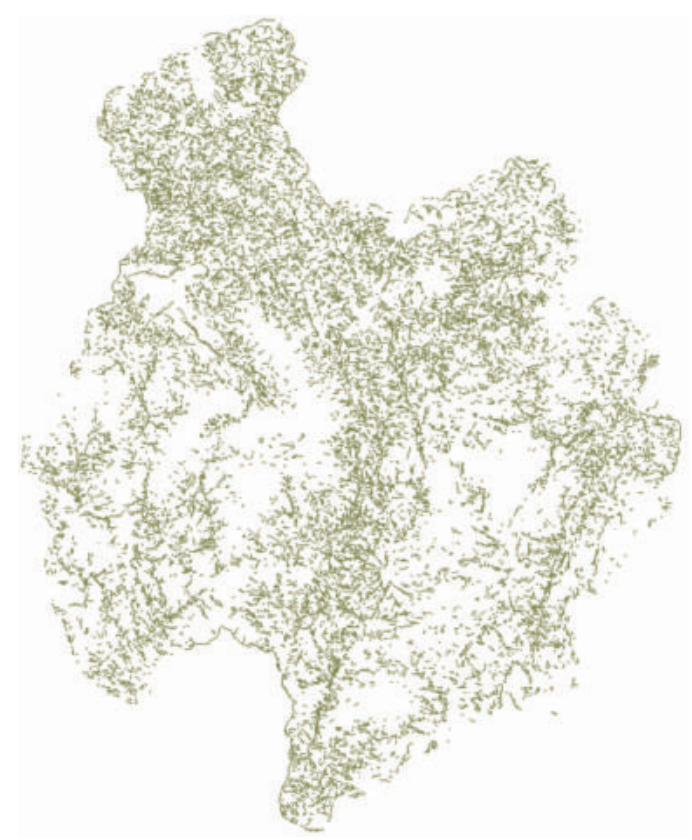

Hướng TB-ĐN

Hình 2: Kết quả tách chiết lineament theo các hướng 
chiết (độ dài, hướng, sự giao cắt). Các kỹ thuật chuẩn hóa được thực hiện trên phần mềm ArcGIS 10.0.

Có một vài dạng lineament lỗi như không kết nối (bởi vì sự gián đoạn của phản xạ phổ trên ảnh), phủ chờm và quá gần nhau (xuất hiện trong hệ thống sông suối hoặc bóng ảnh), các lineament dị thường (quá ngắn hoặc quá dài). (xem hình 3)

Hai lineament, $a, b$, quá gần và cắt nhau. Nếu góc $\alpha$ là đủ nhỏ, cả hai có thể được thay thế bằng một lineament $r$ mới. Chiều dài của $r$ được tính thông qua giá trị lớn nhất và nhỏ nhất của a và $b$. Trong khi hướng của $\mathrm{c}$ được tính như hướng trung bình của $a$ và $b$ (xem hình 3a).

Hình $3 b$ cho thấy một trường hợp ít phổ biến nhưng rất quan trọng. Trong trường hợp này, hai lineament, $a, b$, quá gần và không cắt nhau. Nếu góc $\alpha$ là đủ nhỏ và khoảng cách là nhỏ, vì vậy nó có thể được kết hợp như là $r$. Chiều dài của $r$ được tính thông qua tối đa và tối thiểu phối hợp của a và $b$. Hướng của $r$ được tính từ hướng trung bình của $a$ và $b$. Trường hợp này xảy ra ở địa hình phức tạp với thung lũng thẳng hoặc dòng sông rộng.
Sự phức tạp được thể hiện trong hình. 8c. Trong trường hợp này, chúng ta có một sự kết hợp của các trường hợp trong hình. $3 a$ và $3 b$, các lineament, $a, b$ và $c$, là quá gần. Góc và độ dài được sử dụng để tạo ra một lineament mới

\subsubsection{Chuẩn hóa bằng tay}

Để các lineament giả được loại bỏ trên cơ sở chồng ghép với bản đồ địa hình. Công việc này được xử lý dễ dàng bằng nhiều phần mềm GIS sã̃n có. Trong nghiên cứu này ARCGIS 10.0 đã hỗ trợ rất hữu hiệu cho công việc loại bỏ lineament giả. Bên cạnh đó việc hiển thị các lineament thô trên mô hình số độ cao (DTM) và ảnh vệ tinh trong không gian 3 chiều (3D), cộng với khả năng trợ giúp người sử dụng quan sát các đối tượng theo nhiều hướng khác nhau cũng làm cho công việc loại bỏ lineament giả dễ dàng và chính xác hơn.

Một lỗi khác mà có thể ảnh hưởng đến việc tách chiết lineament là ranh giới của các lưu vực phụ. Để loại bỏ các lỗi này, các DEM của khu vực nghiên cứu được sử dụng để xác định các lưu vực phụ. Tất cả lineament trong một vùng đệm của ranh giới lưu vực phụ được loại bỏ. Bằng cách sử

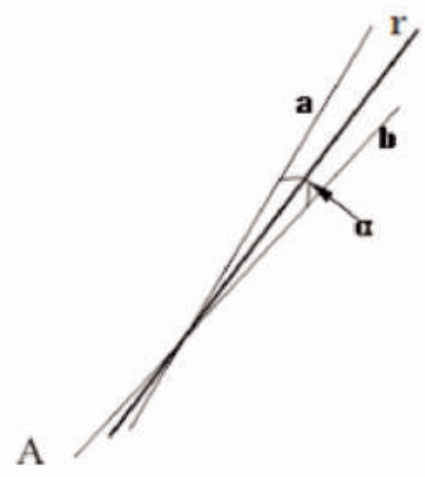

B

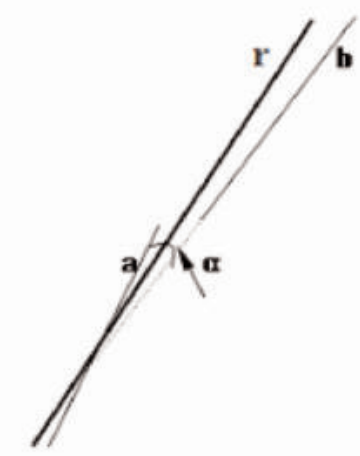

C

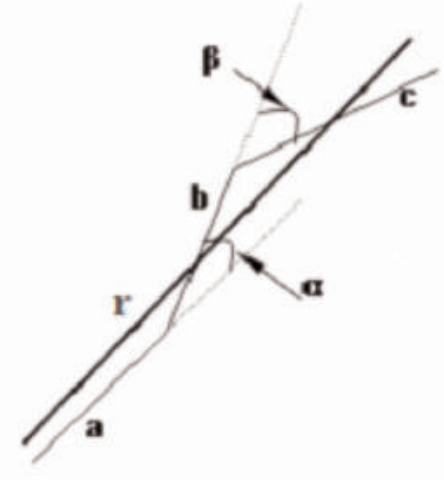

Hình 3: Một số lỗi lineament thường gặp được tự động chuẩn hóa 
dụng các mô tả thống kê của các bản đồ lineament, tất cả các bất thường lineament, chẳng hạn như, quá ngắn hoặc quá dài có thể được gỡ bỏ. Công cụ này rất hữu ích cho việc kết hợp bản đồ lineament từ các nguồn khác nhau, vì mỗi kỹ thuật hoặc loại dữ liệu có thể cung cấp kết quả riêng.

\section{Kết quả}

3049 lineament với độ dài khác nhau giữa $1000 \mathrm{~m}$ và $3992 \mathrm{~m}$ đã được phát hiện trong tổng số (xem hình 4). Phân tích mật độ lineament đã được thực hiện cho một đánh giá mô tả đầu tiên của bản đồ lineament. Rõ ràng là lineament trong các khu vực có mật độ cao có độ dài nhỏ hơn so với những người ở các vùng mật độ thấp hơn. Biểu đồ hoa hồng (xem hình 5) minh họa các hướng lineament, với 2 hướng nổi bật là $Đ B-T N$ và hướng $Đ-T$. Điều này cũng phù hợp với hướng đứt gãy chính của địa hình khu vực nghiên cứu, được tách từ bản đồ địa chất.

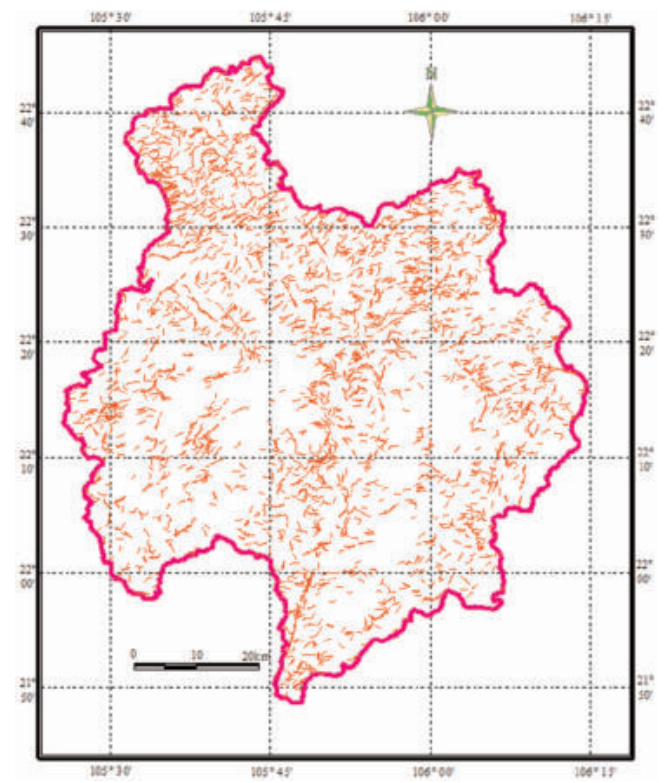

Hình 4: Sơ đồ lineament khu vực nghiên cứu
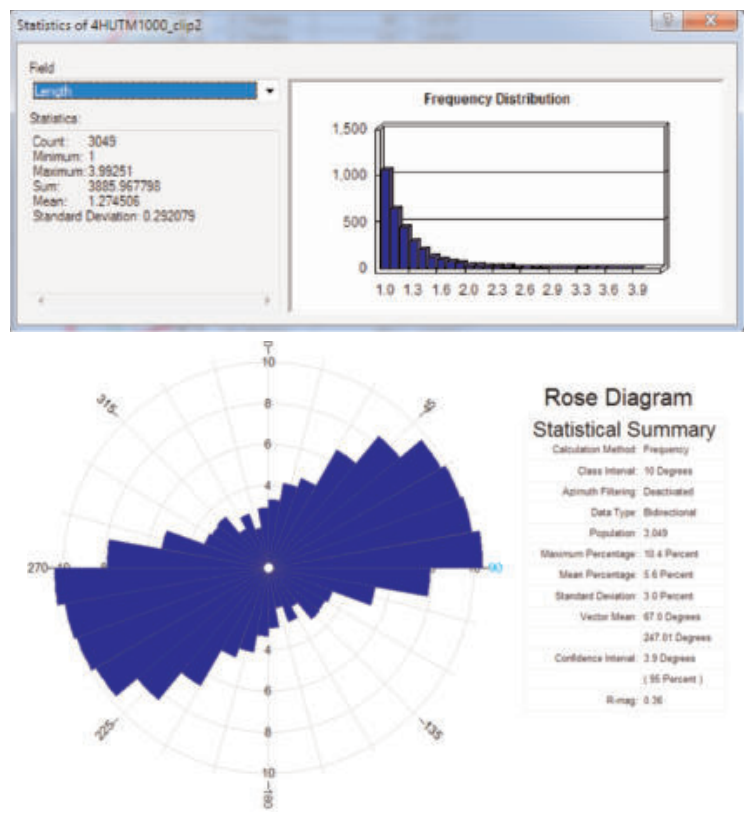

Hình 5: Các thống kê kết quả bản đồ lineament

\section{Thảo luận}

Trong nghiên cứu này các lineament khu vực tỉnh Bắc Kạn được tự động tách chiết bằng module LINE của $\mathrm{PCI}$ Geomatica từ ảnh vệ tinh SPOT. Kỹ thuật lọc theo hướng (tăng cường chất lượng ảnh) đã được áp dụng để tăng cường khả năng phát hiện và tách chiết các đối tượng dạng tuyến theo 4 hướng chính. Để loại bỏ các lineament giả và chuẩn hóa lineament, một số kỹ thuật tự động đã được phân tích. Các kết quả thống kê và biểu đồ hoa hồng chỉ ra hướng lineament trùng với cấu trúc địa chất của khu vực nghiên cứu. Bản đồ lineament có thể được sử dụng cho mục đích phân tích trượt lở. $O$

\section{Tài liệu tham khảo}

[1]. Costa, R. D., Starkey, J., 2001. Photo Lin: a program to identify and analyze linear structures in aerial photographs, satellite images and maps. Comput Geosci 27(5): 527-534.

[2]. Jensen, J. R., 1996. Introductory Digital Image Processing, Prentice Hall 
Series in Geographic Information Science, New Jersey, 316 p.

[3]. Hung, L.Q., Batelaan, O., and De Smedt F., 2005. Lineament extraction and analysis, comparison of LANDSAT ETM and ASTER imagery. Case study: Suoimuoi tropical karst catchment, Vietnam. Remote Sensing for Environmental Monitoring, GIS Applications, and Geology V, edited by Manfred Ehlers, Ulrich Michel, Proc. of SPIE Vol. 5983, 59830T, 0277-786X/05/\$15 - doi: 10.1117/12.627699

[4]. Mostafa, M. E., Bishta, A. Z., 2005. Significance of lineament patterns in rock unit classification and designation: a pilot study on the Gharib-Dara area, northern Eastern Desert, Egypt. Int J Remote Sens 26(7):1463-1475

[5]. O'Leary, D.W., Freidman, J.D., Pohn, H.A., 1976. Lineaments, linear, lineationsome proposed new standards for old terms", Geological Society of America Bulletin 87, 1463-1469.

[6]. PCI Geomatica Users' Manual, 2001.

[7]. Rowan, L.C., Lathram, E.H., 1980.
Chapter 17 Mineral Exploration. In Remote Sensing in Geology; Siegal, B.S., Gillespie, A.R., Eds.; John Wiley and sons: New York, NY, USA, pp. 553-605.

[8]. Sarp, G., 2005. Lineament Analysis From Satellite Images, North-West Of Ankara, Msc thesis, Middle East Technical University, $76 \mathrm{p}$

[9]. Stefouli, M., Angellopoulos, A., Perantonis, S., Vassilas, N., Ambazis, N., Charou, E., 1996. Integrated analysis and use of remotely sensed data for the seismic risk assessment of the southwest Peloponessus Greece. First Congress of the Balkan Geophysical Society, 23-27 September, Athens, Greece

[10]. Süzen, M.L. and Toprak, V., 1998. Filtering of Satellite Images in Geological Lineament Analyses: An Application to a Fault Zone i n Central Turkey, International Journal of Remote Sensing, 19(19), 11011114.0

\section{Summary}

\section{Automatic lineament extraction from SPOT imagery for Bac Kan area}

MSc. Nguyen Dinh Tai, Assoc. Prof. Dr. Nguyen Ngoc Thach, Faculty of Geography, VNU University of Science

This research presented automatic lineaments extraction method from SPOT satellite imagery for the purpose of landslide risk assessment. The techniques of image enhancement have been used to improve the ability to detect the lineament according to different directions. LINE Module in PCI Geomatica software was used to automatically extract lineaments according to predetermined parameters. The standardization of lineaments, as well as removing "error" lineament have be done by using some algorithms and other verified data. The statistical results demonstrated lineaments obtained by automatically extraction method from satellite images accordance with the direction structure terrrain of the study area. $O$

Ngày nhận bài: 20/01/2015. 